NASA Technical Memorandum 102048

\title{
A Vector Scanning Processing Technique for Pulsed Laser Velocimetry
}

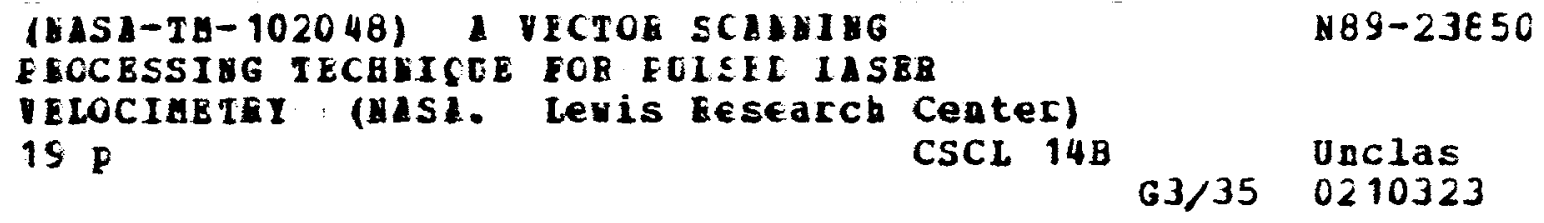

Mark P. Wernet

Lewis Research Center

Cleveland, Ohio

and

Robert V. Edwards

Case Western Reserve University

Cleveland, Ohio

Prepared for the

13th International Congress on Instrumentation in Aerospace Simulation Facilities (ICIASF) sponsored by the Institute of Electrical and Electronics Engineers Göttingen, West Germany, September 18-21, 1989

\section{Nons}




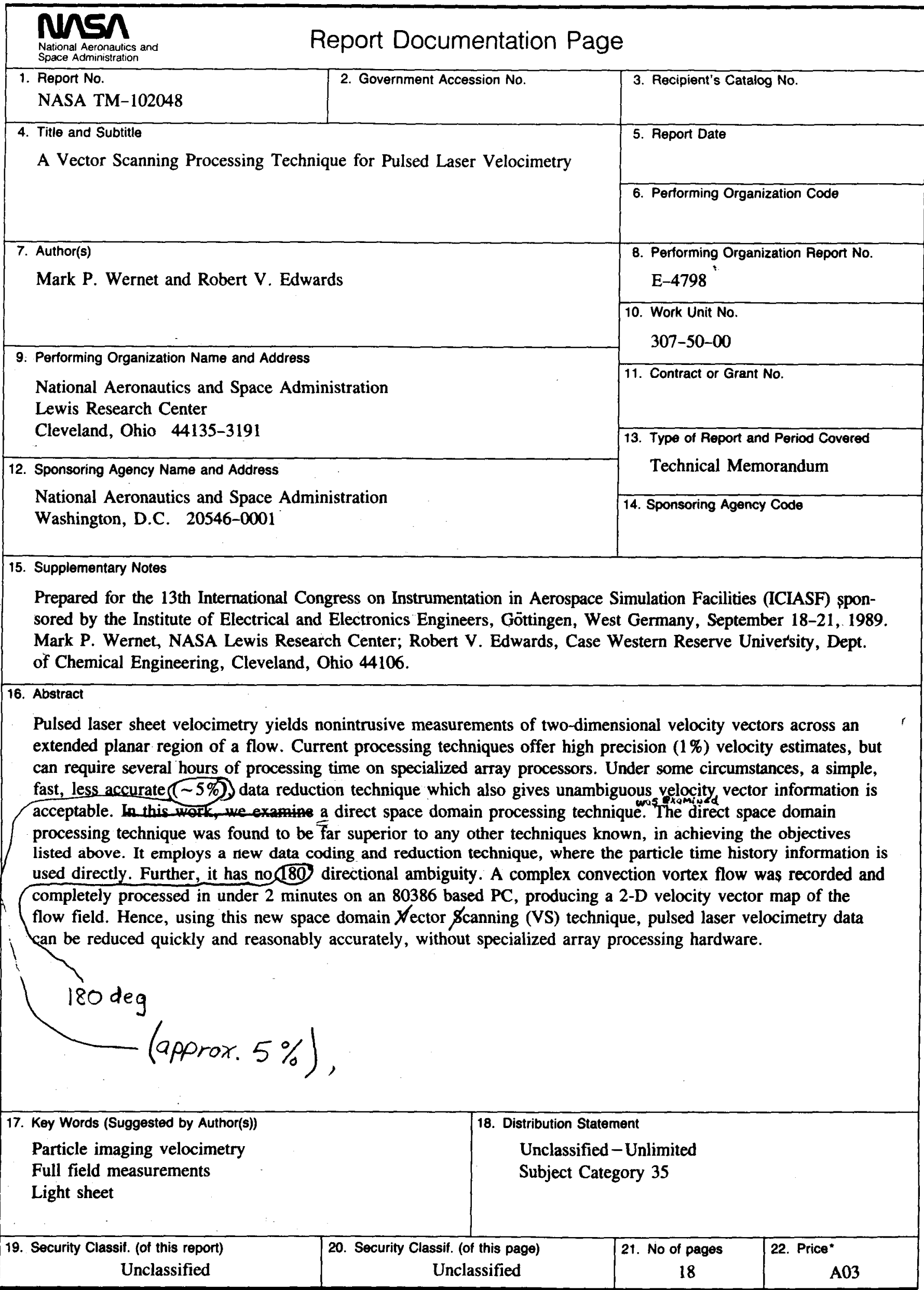




\author{
A VECTOR SCANNING PROCESSING TECHNIQUE \\ FOR PULSED LASER VELOCIMETRY \\ by \\ Mark P. Wernet \\ National Aeronautics and Space Administration \\ Lewis Research Center \\ Cleveland, Ohio 44135 \\ and \\ Robert V. Edwards \\ Case Western Reserve University \\ Dept. of Chemical Engineering \\ Cleveland, Ohio 44106
}

\begin{abstract}
Pulsed laser sheet velocimetry yields non-intrusive measurements of two-dimensional velocity vectors across an extended planar region of a flow. Current processing techniques offer high precision (1\%) velocity estimates, but can require several hours of processing time on specialized array processors. Under some circumstances, a simple. fast, less accurate ( $\sim 5 \%)$, data reduction technique, which also gives unambiguous velocity vector information is acceptable. In this work, we examine a direct space domain processing technique. The direct space domain processing technique was found to be far superior to any other techniques known, in achieving the objectives listed above. It employs a new data coding and reduction technique, where the particle time history information is used directly. Further, it has no $180^{\circ}$ directional ambiguity. A complex convection vortex flow was recorded and completely processed in under 2 minutes on an 80386 based PC, producing a 2-D velocity vector map of the flow field. Hence, using this new space domain Vector Scanning (VS) technique, pulsed laser velocimetry data can be reduced quickly and reasonably accurately, without specialized array processing hardware.
\end{abstract}

\section{INTRODUCTION}

Pulsed laser sheet velocimetry yields non-intrusive measurements of velocity vectors across an extended 2 -dimensional region of the flow. Double pulsing a laser light sheet perpendicular to the direction of observation yields a record of the particle positions at two instances of time over the entire plane. The number density and size of particles in the fluid determine the data analysis approach. For a large number density of recorded particle images, the Laser Speckle Velocimetry (LSV) data analysis procedure is used $[1,2]$. In LSV, the recorded particle photographs are usually analyzed by illuminating small regions of the photograph by a low power laser beam. The far field diffraction pattern of the illumination beam contains speckly Young's fringes. The fringes are oriented perpendicular to the local velocity vector, and the fringe spacing is inversely proportional to the velocity vector magnitude. Measurement of the fringe spacing and orientation gives the local velocity vector. Accuracies as high as $1 \%$ are possible with the LSV technique [1]. By repeating the process over a grid of points on the transparency, a 2-D velocity vector map is constructed of a plane in the flow. For low number density double pulsed photographs, different processing schemes are applied. These low number density processing techniques are referred to as Particle Imaging Velocimetry (PIV). Due to the low particle density, individual particle displacement vectors can be determined, instead of the average displacement of a group of particles as in laser speckle. Particle tracking algorithms employing image processing techniques can be used to reduce the recorded PIV transparencies. Alternatively, the point-wise laser beam readout technique can be used to determine the local velocity vectors. Hence, different processing schemes may be applied depending on the recorded particle number density.

The point-wise laser beam readout of a recorded particle image transparency is a tedious and time consuming process. In their present state, both PIV and LSV data reduction techniques are similar to the point-wise measurements obtained using laser anemometry, which are time consuming and have positioning constraints. For a typical $100 \mathrm{~cm}^{2}$ region of a transparency analyzed on a $1 \mathrm{~mm}$ grid, there are on the order of $10^{4}$ points to be analyzed. At each point, the processor must determine the fringe pattern spacing and orientation. This exhausting data analysis technique is most efficiently handled by an automated procedure. However, even an automated procedure employing specialized array processors can still require several hours of processing time.

For all of the effort that has been expended to analyze particle image transparencies and determine the fringe spacing and orientation at $\sim 10^{4}$ points, there still exists a $180^{\circ}$ directional ambiguity. The fringe patterns generated by the readout beam are symmetric, hence, the velocity vector is known only to $\pm 180^{\circ}$. More information must be included at the recording stage of the process to resolve the direction unambiguously $[3,4]$.

Under some circumstances, a simple, fast, less accurate $(\sim 5 \%)$, data reduction technique, which also gives unambiguous velocity vector direction information is acceptable. In this work. we describe a new and fast data reduction technique for Pulsed Laser Velocimetry data. A simplified convolution algorithm was developed which uses the time history information of the recorded particle images. The particle image data is directly recorded using a CCD camera. The new Vector Scanning (VS) technique runs on a high performance $\mathrm{PC}$ and obtains an effective processing rate of 580,000 velocity 
vector convolutions/second. The VS technique provides reasonably accurate estimates $(2-18 \%)$, where the accuracy is a function of the particle displacement between exposures. Using the particle time history information, unambiguous velocity vector information can be determined. Further, 5-pulse exposures produced high quality data with few false identifications.

Data from a complex convection vortex flow was processed using the new technique. The convection vortices contained a wide dynamic range in velocity magnitude and a full $360^{\circ}$ flow angle variation. The recorded frames contained over 350 particle images per exposure. The VS technique yielded good results from this complex flow field. The Vector Scanning technique should work equally well in air flows. Current research efforts are concentrating on applying the new Vector Scanning technique to moderate speed air flows. The use of gated, intensified CCD cameras are being explored for integration into a high framing rate electronic recording system, which will enable measurement of higher speed flows.

A new, state of the art, $\mathrm{PC}$ based, pulsed laser velocimetry data reduction system has been constructed and tested. A complex flow field can be recorded and completely processed in under 2 minutes, producing a $2-D$ vector map of the flow field.

\section{VECTOR SCANNING PROCESSING TECHNIQUE}

The Vector Scanning processing technique to be described here involves two distinct steps: the data acquisition and centroid processing step; and the velocity vector scanning step; see figure 1. The Vector Scanning processing technique can easily be implemented on an existing pulsed laser velocimetry setup. The flow system is configured to record the discrete particle images (the PIV particle density regime). The seeded flow field to be recorded is illuminated by a light sheet. The essential steps in the new procedure are:

1) The images of the particles are recorded on separate records for each exposure. The recorded particle image frames are separated in time by a preselected sequence of times, $\Delta \mathrm{t}_{1}, \Delta \mathrm{t}_{2}$, etc.

2) The particle images are converted to single pixel centroid positions by an expeditious approximation routine. This routine will be discussed in detail below.

3) The recorded frames are scanned for a sequence of preselected velocity vectors using a rapid, logical cross-correlation routine.

For purposes of illustration, consider the particle image record made by recording the particle positions at two instances of time on separate frames. After the particle centroids are computed, a velocity vector to be scanned for is selected. Then the first processed frame is scanned for a particle image. When a particle is found, the second frame is checked for a particle image corresponding to the position of the first particle image if the recorded particle velocity was the preselected one. If there is a particle image there, that velocity is recorded at the point and both images are deleted from the data frames. The program then continues to scan the rest of the frame for that velocity until all particle positions in the first frame are exhausted. A new velocity vector is then selected and the scanning procedure repeated until the entire preset velocity range is covered. Note that the only arithmetic steps required are logical, with no additions or multiplications required. Thus the procedure is computationally very efficient. Note also that there is no $180^{\circ}$ directional ambiguity because we know which is the first image and which is the second.

In practice, the images from different times are places on the same record, but are distinguished from one another by the method with which the particle positions are encoded. However, to understand the method, it is best to think of the processing as taking place using a sequence of separate frames.

Actually it is better to use more than a pair of images. Using more than two images allows you to examine a higher dynamic range in velocity and reduces the probabilities for error. The images can be recorded on individual photographic plates, or directly onto a CCD imaging camera. Direct digital recording on a CCD provides instantaneous data quality verification and fast data reduction turnaround times. In either case, the data must be preprocessed in the computer. The preprocessing on the computer involves the determination of all of the recorded particle image centroids. The centroid processing produces a particle image field map of single pixel particles, of equal size and amplitude. A multiple exposure, time history, single pixel particle map can be generated by processing several successive frames, and coding the single pixel particle amplitudes according to the frame number in the sequence. These digitally preprocessed time history, pixel particle maps are the input frames to the Vector Scanning processing program.

Digitally processing the particle images to determine their centroids introduces quantization errors. Although the centroids can be calculated to sub pixel accuracy, the discrete image only permits the representation of a particle centroid to within a pixel. The quantization error is minimized by using high resolution $(512 \times 512$ pixel) CCD arrays, but is also a function of the imaged particle size, and image quality. A Maximum Likelihood Estimation (MLE) analysis [5] was performed to examine the relationship between these parameters. The MLE analysis represents the best estimate (lowest variance) that can be obtained from the modeled system. The details of the analysis are given in reference 6 .

Using the results of the MLE analysis, and assuming that the largest recorded particle image will cover 5 pixels, the largest error in estimating the particle image centroid will be approximately $\pm \frac{1}{2}$ pixel. Hence, the rms error in determining a particle displacement between two images will be $\pm 1 / \sqrt{2}$ pixels. The total particle displacement between the first to last exposures can be on the order of 4 to 40 pixels, which gives a relative error in the velocity of 18 to $2 \%$, respectively. The angular error in the detected velocity vector is also a function of the particle displacement. The error in determining the vector angle was determined to be equal to the arctangent of the total displacement relative error. 
The individual particle images which were recorded in the multiple exposures make up a particle displacement pattern. The number of images and their spacing is determined by the number of recorded exposures and the time between exposures. The optimum choice of these parameters depends on the dynamic range of the flow field under study. For our purposes, a 5-pulse exposure will be assumed, since the data were recorded in this format. For a 5-pulse light sheet exposure, there are 5 particle images which can be used to define a velocity vector. The Vector Scanning technique searches for these particle displacement patterns (velocity vectors). A convolution procedure could be used to perform a search for these particle displacement patterns. In a convolution search operation, an image frame is convoluted with a mask. When the image contains the mask, a large amplitude peak results. The amplitude of the peak represents the degree of matching. The mask is convolved with the entire image, producing peaks which denote the existence and location of the mask in the image frame. A similar technique is used in the Vector Scanning algorithm, where the convolution mask is the particle displacement pattern.

The Vector Scanning processing program operates on the preprocessed time history frame. The processing program picks a velocity vector magnitude and angle to be searched. The displacement vector containing the relative particle positions which must be satisfied is generated. The program starts at the upper left corner of the data frame to be processed, offset from the edges so that the vector pattern does not exceed the bounds of the array. The initial search location on the data frame is selected, which corresponds to the initial particle position in the generated displacement vector. In order for the convolution to detect a velocity vector, each value in the displacement vector pattern must match the values at the corresponding locations in the data frame. Usually, in a convolution calculation, the test pattern is multiplied by the coincident points in the data frame, and the products summed. For a 5-pulse exposure, we would expect 5 non-zero products, and a result equal to the sum of the five products. This is how the convolution works, but we do not have to follow these rules strictly. Instead of multiplying and summing, the program performs a logical comparison. The coordinates to be checked are those in the generated displacement vector. These are the 5 locations that we expect to find non-zero pixel values, (or particles), for the selected velocity vector. The program merely checks to see if the values in the data frame are non-zero. If any of the 5 coordinates checked are zero, the check ends, and the program moves to the next location on the data frame. If all five locations are non-zero, then a correlated result has been found. The vector coordinates are saved along with the current search vector magnitude and angle. Using this search procedure, the data frame is convoluted with the test pattern (the particle displacement vector). No multiplications or additions are required, just logical comparisons.

Now that a rough outline of the program has been presented, we can refine the algorithm, building in some intelligence. The time history information encoded into the data frame will reduce the chances of a false vector identification, and reduce the program run time. The time history was encoded in the pixel amplitudes. Particle images found in frame \#1 were assigned an amplitude of $2^{1}$. Particles from frame \#2 were given amplitudes $2^{2}$, and so on. Before initiating the displacement vector search, the data frame is scanned and the coordinates of all of the pixels with amplitude equal to $2^{1}$ are stored. These are all of the particle positions on the first frame, which are the starting points for all of the displacement vector checks. Using these stored coordinates eliminates the search over the entire data frame. The program knows where the starting particle locations are, and only searches at these locations. This simplification greatly reduces the program search time per velocity displacement vector. A flow chart for the program is given in figure 2 .

The program searches for each successive particle in the 5-pulse record on a successive frame in the exposure sequence. When a null condition is encountered the program moves to the next particle position found from the first exposure. When a vector is found, the vector attributes are stored, and then the pixel locations of the detected displacement vector are set to zero. Zeroing the pixel values eliminates the chance of falsely identifying a particle from the currently identified velocity vector as being part of another velocity displacement vector on a subsequent vector search.

The pixel registration error from , he particle centroid processing can also be accommodated by the new search procedure. The registration error was defined as $\pm \frac{1}{2}$ pixel. in both dimensions. For the program to accommodate the registration error, a larger region must be defined for the permitted positions of the $2^{\text {nd }}$ through $5^{\text {th }}$ particle positions. The displacement vector search pattern used for accommodating the pixel registration error is shown below:

\begin{tabular}{|c|c|c|c|c|}
\hline 1 & $2^{\text {nd }}$ & $3^{\text {rd }}$ & $4^{\text {th }}$ & $\bar{j}^{\text {th }}$ \\
\hline & बQ & वृD & व०ם & QDQ \\
\hline & $\square \bullet \square$ & $\square \bullet \square$ & $\square \bullet \square$ & $\square \bullet \square$ \\
\hline & प्र० & סטव & व०० & סם \\
\hline
\end{tabular}

The exposure numbers are indicated above the pattern. The solid dots indicate the original displacement vector pattern. The hollow squares represent both the \pm 1 pixel searched in the vector displacement direction and the \pm 1 pixel lateral search. The lateral pixel search accounts for stray pixels not lying in a straight line. The lateral pixel search is also performed at \pm 1 pixel in the vector displacement direction. The initial displacement point is a single position, we know this position exactly from the particle position search performed on the first exposure frame. During the search, the appropriate pixel value must occur in each of the $3 \times 3$ pixel regions for a velocity vector to be determined. When a vector is found. the program then looks inside each of the regions to find the exact location of the detected pixels. The exact pixel locations are used to calculate the particle displacements between exposures. The actual particle displacements are stored. 'The three pixel wide search area accounts for lateral displacements, but sacrifices the angular resolution. This angular 'slop' is corrected by determining the actual location of the $5^{\text {th }}$ pixel in the $3 \times 3$ region. If the detected pixel is in the row containing the solid dots, the angle remains the same. If the detected pixel is in one of the rows with all hollow squares, the recorded angle is incremented or decremented by the arctangent of 1 over the sum of the displacements. The angular correction maintains the precision of the technique to $\pm \frac{1}{2}$ pixel in the lateral direction. 
The optimized vector search algorithm uses the time history information, and accounts for the pixel registration error without compromising the accuracy of the estimate or increasing the CPU time. The 5-pulse exposure sequence is not required for directional ambiguity removal when using the time history information. Only 2 exposures are really needed to obtain unambiguous direction information when the particle time histories are available and the data density is low. For higher data densities, more than two exposures are required to minimize the error rate. A false identification occurs when independent particle images are misconstrued as originating from a single particle. The 5-pulse exposure gives us 5 samples of the flow, which reduces the false identification rate. The false identification rate is dependent on the particle number density and CCD array resolution. The error rate decreases as the number of frames used in the processing technique increases.

The actual processing algorithm required depends on the sampling rate and the characteristics of the flow. Secondary passes through the data frame sequence can be used to increase the available dynamic range, account for curved trajectories, or other peculiarities of the system under study. Also, the processing algorithm could be designed to "learn" about the flow field as the velocity vectors are detected. The data obtained from several exposures is of good quality, and could be used as a training set for subsequent searches through the data for incomplete displacement pattern records or for slower velocities.

The dynamic range of the VS technique is limited by the maximum particle displacement between exposures. The minimum displacement between exposures is 1 pixel. The maximum displacement between exposures is typically 10 pixels. Thus, the total particle displacement is 40 pixels for a 5 exposure record. Attempting to track particles over larger displacements results in more false identifications than successful measurements. Hence, using 5 equally spaced exposures yields a 10:1 dynamic range in velocity magnitude.

The dynamic range of the VS processing technique can be extended by using variable inter-exposure times. The fastest particle velocities are recorded using the shortest inter-frame time. However, low velocity particles may not have been displaced by a discernible amount during these short inter-frame times. A slower framing rate, which overlaps the high framing rate sequence, can be used to extend the velocity sensitivity range. Figure 3 shows two overlapping exposure sequences of recorded frames. The series 1 sequence uses an inter-exposure time of $\Delta \mathrm{T}$, which corresponds to the minimum inter-exposure time capability of the recording system. Series 1 is centered about frame \#5. The series 2 sequence uses an inter-frame time of $3 \cdot \Delta T$, and is also centered about frame \#5. Both exposure sequences use frame \#5. Series 1 will be used to detect fast velocities, and series 2 will be used to measure low velocity particles. The results from each series can then be combined to produce a full vector map of higher dynamic range than could be obtained from a singe exposure sequence.

\section{EXPERIMENTAL SETUP}

\section{Surface Tension Driven Convection Flow Svstem}

A complex flow field experimental setup was obtained opportunistically. Discussions with people in the Space Experiments Division at NASA Lewis Research Center revealed an on-going PIV study of a future space experiment on Surface Tension Driven Convection (STDC) flows. STDC flows affect crystal growths in a zero gravity environment [7]: In the zero gravity environment, the surface tension forces usually suppressed by the gravity forces become the dominant effects in heated flows with free surfaces. The STDC program is in an evaluation stage where the best means of reducing PIV data obtained from the system are being analyzed. Video tapes of the flow field pattern were available for analysis. We did not have complete control over the illumination and recording parameters. so the measurements could not be optimized. However, a good test of the applicability of the Vector Scanning method was obtained.

By using a circularly symmetric reservoir, and heating the free surface at the center, a radial surface flow was generated. The walls of the reservoir turned the radial flow downwards. The warmer fluid flowing down the walls of the reservoir then rises due to convection. establishing the vortex cells.

The STDC system consisted of a square plexiglas block with a cylindrical well bored into the center as the test cell. A cylindrical casing was also glued on top of the block; see figure 4 . The faces of the plexiglas were highly polished optical quality surfaces. The external dimensions of the block were $152.4 \times 152.4 \times 50 \mathrm{~mm}$. The cylindrical well inside the block was $102 \mathrm{~mm}$ in diameter and $45 \mathrm{~mm}$ deep. The plexiglas tube on the top of the block matched the cylindrical well inside diameter and was $600 \mathrm{~mm}$ high.

The cylindrical well was filled with fluid up to the block/tube interface. The fluid was Dow Corning 200 Fluid (silicone oil) of 10 centistoke kinematic viscosity. The fluid index of refraction, 1.4, was matched to the refractive index of the plexiglas test cell. The matched indices of refraction eliminated any astigmatic effects caused by the inner cylindrical reservoir. The silicone oil was seeded with $3 \mu \mathrm{m}$ diameter $\mathrm{Al}_{2} \mathrm{O}_{3}$ particles. The number density of the particles was on the order of 2000 particles $/ \mathrm{ml}$ of silicone oil. The large density difference between the seed particles and the fluid resulted in a large settling velocity. The system was stirred occasionally to resuspend the particles.

The external heating source used to drive the convection driven flow was an 8 Watt $\mathrm{RF}$ excited $\mathrm{CO}_{2}$ laser. The $\mathrm{CO}_{2}$ laser operated at a wavelength of $10.6 \mu \mathrm{m}$, which was completely absorbed by the silicone oil within $0.2 \mathrm{~mm}$ of the surface. The $\mathrm{CO}_{2}$ laser beam was delivered to the surface at normal incidence. The size of the beam delivered to the fluid surface was approximately $5 \mathrm{~mm}$ in diameter. 
The laser light sheet for particle illumination was supplied by a .5mW HeNe laser. The light sheet was generated by a pair of cylindrical lenses and was incident on the fluid surface in the same plane as the $\mathrm{CO}_{2}$ heating laser beam. The HeNe light sheet illuminated the entire cross section of the cylindrical reservoir.

A Silicon Intensified Target (SIT) camera provided a standard RS-170 video output signal of the counter rotating flow cells. A frame grabber board was used to grab the images from the SIT camera. The inter-frame times could be selected in multiples of $154 \pm 1$ millisecond. This slow framing rate is a limitation inherent to the frame-grabber board. The SIT camera was oriented perpendicular to the plane of the light sheet.

Measurements of the small seed particles was possible with the low $(5 \mathrm{~mW})$ source illumination through the use of the SIT camera.

\section{Particle Centroid Processing}

The particle image data was obtained using SIT camera with the frame-grabber board. The frames contained $512 \times 488$ elements with 256 grey levels. The individual frames from each exposure in the sequence were available for independent processing. A particle boundary detection and centroid calculation program was written in Fortran 77 on a Compaq 38625 Mhz computer.

The boundary detection program sequentially processed all of the frames in the exposure sequence, and summed the results into a single processed frame. The frames to be processed were compared to a threshold level as they were read into the computer memory. The threshold level was set just high enough to remove the background noise from the camera. The boundary program initiated the search in the upper left corner of the frame, searching from left to right for a non-zero pixel value. When a non-zero pixel value was detected, the program searched the particle image boundary in a clockwise manner.

The program scanned the perimeter coordinates to determine the particle image minimum $\mathrm{x}$ and $\mathrm{y}$ extents, and the maximum $x$ and $y$ extents. These limits were used to calculate the particle image centroid. A rectangular area delimited by the minima and maxima found above was integrated along the $x$ and then the $y$ axes independently to obtain the $x$ - and $y$-projections of the particle image. The particle image's mean $x$ and mean $y$ values were calculated from the $x-$ and $y$-projections respectively. The particle image centroid coordinates, and the current frame number being processed were stored. The rectangular area delimited by the $\mathrm{x}$ and $\mathrm{y}$ minima and maxima was then set to zero. Setting these pixel values to zero avoided redetecting the particle image on a subsequent pass. The program then resumed searching in a left to right direction for the next non-zero pixel value.

The processed image data was then written to an image file, where the particle positions were encoded into a single pixel, and the amplitude of the particle image pixel indicated from which frame out of the multiple pulse exposure sequence it was detected. The processed output frame occupied the same amount of memory as the individually processed input frames, $512 \times 512 \times 1$-byte. or $256 \mathrm{~K}$ bytes of memory.

\section{RESULTS AND DISCUSSION}

\section{Surface Tension Driven Convection Data}

Particle image data were obtained from the STDC video tape. Extended velocity vector magnitude sensitivity was achieved by using a variable inter-frame time 9 exposure sequence as discussed previously and illustrated in figure 3 . The $\Delta \mathrm{T}$ for the series 1 exposure sequence was 154 milliseconds. The series 2 inter-frame time was $3 \cdot \Delta \mathrm{T}$, or 462 milliseconds.

The $\mathrm{CO}_{2}$ laser beam power delivered to the surface was adjusted so that particle image patterns were easily observed on the sum of the collected data frames. The $\mathrm{CO}_{2}$ laser operating power was approximately $1 \mathrm{Watt}$. At this heating rate, the flow convection cells were fully developed. and centered about the incidence point of the $\mathrm{CO}_{2}$ laser beam. The convection cells, once fully developed are usually isolated from the bulk fluid.

A single raw data frame from the 9 exposure sequence is shown in figure 5 . The dithering program used to generate this 16 grey level image is of limited quality. Only the brightest particle images are readily observed in the figure. Figure 5 shows a large amount of flare light from the edges of the reservoir, and from the screw studs in the base of the test cell. The recording parameters were not optimal for this video taped data. The flare from the edges was eliminated by entering the coordinates of the window containing the particle images to the centroid processing program. For more optimal results, the flare light should be eliminated. the contrast ratio of the particle images to the background should be increased, and the recorded image should fill the field of view.

The raw data frames were processed using the centroid processing program. The processing threshold level was 150 grey levels. The particle centroid processing time was approximately 3 seconds per frame. The series 1 data set was constructed using frame numbers $3,4,5,6$, 7 . Series 2 was generated using frame numbers $1,2,5,8$, 9. The centroid processed data frames for series 1 and series 2 are shown in figures 6 and 7 respectively. Symbols were used to represent the detected particle image centroids. There are 5 different symbols used in figures 6 and 7 for representing the time history information:

\section{Particles from exposure \#1: Particles from exposure \#2: + Particles from exposure \#3: 0 Particles from exposure \#4: Particles from exposure \#:5: $\times$}

There were 1899 particles detected in series 1 and 1814 particles detected in series 2 . The spacing between independent particle images records is on the order of, or less than the total particle displacements from the first to last exposure in the series. Hence, the data density is 
slightly higher than the PIV regime of particle seed density.

A scale frame was required in order to obtain a pixel to length conversion factor. A ruler was placed in the plane of the light sheet and recorded. The measured scale was 164 pixels $/ 63.5 \mathrm{~mm}$, or $387.2 \mu \mathrm{m} /$ pixel. Using the scale factor, the size of the imaged flow field is approximately $200 \times 200 \mathrm{~mm}$.

The data frames series were processed using the Vector Scanning technique. The velocity vector magnitude and angular searches were from 1 to 9 pixels and $360^{\circ}$ respectively. Figure 8 shows the velocity vector map for the series 1 data set. The processing time was 34 seconds, and 3346 velocity vector displacement patterns were searched. The processing time scales with the number of recorded particle images. The total number of vectors detected was 99 . The velocity vector map in figure 8 shows the fully developed convection cells. There are a few falsely identified velocity vectors in the vector map. The velocity is highest in the region between the two counter rotating convection cells. The inter-frame time was too long to detect the particle velocities in this region. The maximum detected velocity was $20.5 \mathrm{~mm} / \mathrm{s}$, with a relative uncertainty of $2.1 \%$. The angular error in the maximum velocity was $\pm 1.2^{\circ}$. The minimum measured velocity was $2.5 \mathrm{~mm} / \mathrm{s}$, with a relative error of $17.6 \%$. The angular error in the minimum velocity was $\pm 9.9^{\circ}$. A vector magnitude dynamic range of $8: 1$ was measured in this frame series.

Figure 9 shows the velocity vector map for the series 2 data frame. The VS processing time was 38 seconds, and the total number of detected velocity vectors was 82 . The series 2 detected velocity vectors are in the outer edges of the convection cells, where the particle velocities are low. The maximum velocity measured was $8.1 \mathrm{~mm} / \mathrm{s}$ with a relative error of $1.3 \%$. The minimum measured velocity was $0.82 \mathrm{~mm} / \mathrm{s}$, with a relative uncertainty of $17.6 \%$. The maximum and minimum measured velocity vector angular errors were $\pm 1^{\circ}$ and $\pm 9.9^{\circ}$ respectively. A dynamic range of 10:1 has been measured in this data frame series.

The two velocity vector maps show the two regimes of vector magnitudes in the STDC flow field. The series 1 vector map shows the high velocity core region near the heating zone. The lower velocity flow, near the edges of the reservoir, and in the lower portion of the vortex cells, are observed in figure 9 . Both figures show the vector magnitudes proportional to the pixel displacement. Normalization of the vector magnitudes by the inter-frame exposure times used to record each series is required to properly scale the vector magnitudes.

There is a region of overlap in the detected velocity vectors from series 1 and 2 . The velocity vectors detected in series 2, which are common to series 1, have traversed more pixels due to the longer inter-frame time used to record the series 2 data set. Hence, the common vectors from series 2 are more precise in magnitude and direction than those from series 1 .
The two series can be combined to produce a higher dynamic range velocity vector map. When common vectors between the two series are found, the vectors from series 2 will be used, due to their higher precision. The total number of velocity vectors in the combined velocity vector map is 181 . However, after scanning to find common vectors, only 145 unique velocity vectors were found. The combined velocity vector map is shown in figure 10. The vector magnitudes have been scaled by their respective inter-frame times. The dynamic range of the combined results is $25: 1$. The combined figure gives a good indication of the complex flow pattern occurring in the STDC system.

\section{Gaussian Smoothed Velocity Vector Maps}

The randomly sampled data can be made to appear more regular by interpolating the data over a uniform grid of points. A Gaussian smoothing program was used to smooth the combined velocity vector map. The Gaussian smoothing operation calculated the average velocity vector at a select number of grid points. The number and spacing of the grid points were selected by the user. The Gaussian average velocity vector is calculated according to:

$$
\vec{V}(X, Y)=\frac{\sum_{i} \vec{V}\left(X_{i}, Y_{i}\right) \cdot e^{-\left\{\left(X-X_{i}\right)^{2}+\left(Y-Y_{i}\right)^{2}\right\} / 2 \sigma_{\mathrm{g}}^{2}}}{\sum_{i} e^{-\left\{\left(X-X_{i}\right)^{2}+\left(Y-Y_{i}\right)^{2}\right\} / 2 \sigma_{g}^{2}}}
$$

where: $\quad \vec{V}\left(\mathrm{X}_{\mathrm{i}}, \mathrm{Y}_{\mathrm{i}}\right)$ are the detected velocity vectors at the random locations $\mathrm{X}_{\mathrm{i}}, \mathrm{Y}_{\mathrm{j}}$.

$\vec{V}(X, Y)$ are the Gaussian averaged velocity vectors at the $X, Y$ grid points $\sigma_{\mathrm{g}}$ is the user selected Gaussian window standard deviation

All of the velocity vectors in the vector map are used in calculating the average vector at each grid point. When the calculated average vector magnitude is less than 1.0 . no vector is drawn. Hence, smoothed data vectors are only drawn where the data exist.

The Gaussian smoothed data frame is shown in figure 11. Figure 11 was smoothed using $32 \times 32$ grid points, and a Gaussian window standard deviation $\sigma_{\mathrm{g}}$ of 20 . The smoothed data show the general flow structures contained in the randomly sampled data.

The Vector Scanning technique has exhibited good performance, even in relatively high particle concentration flows. The measured data quality was very good, there were few false identifications. The total processing time was 99 seconds, including centroid detection and VS processing. The Vector Scanning technique appears to have the properties sought in a data reduction technique: simple recording and preprocessing 
requirements, fast data reduction times on a $25 \mathrm{Mhz}$ Compaq 386 , and reasonable accuracy (2-18\%).

The Vector Scanning technique achieved an enormous processing rate on a PC. The VS technique used a search region containing one single pixel point, and four $3 \times 3$ pixel search regions. At least one particle was required to be in each search region for a valid velocity vector detection. The detected particle image centroid in each $3 \times 3$ pixel search region could be in any of the nine pixels. The total number of unique velocity vector particle displacement patterns that could be identified using these four $3 \times 3$ pixel search regions was $9^{4}$, or 6561 . Hence, for each search region defined by the scanning program, there are 6561 possible displacement pattern combinations which could be detected. Using the longest processing time above of 38 seconds for 3346 patterns, which each contain four $3 \times 3$ pixel regions, the effective processing rate for the Vector Scanning technique on a $\mathrm{PC}$ is approximately 580,000 unique particle displacement pattern convolutions per second. This is a substantial increase over any of the current state of the art pulsed laser velocimetry processing techniques. The new technique not only gives unambiguous velocity vector direction information, but also achieves a very high processing rate, without the use of specialized array processors.

However, there are some limitations to the Vector Scanning technique. The recorded particle images must have been displaced by at least one pixel between exposures. This is the minimum measurable velocity magnitude. A restriction must also be placed on the maximum particle trajectory curvature. In the current Vector Scanning processing program, the particle displacement patterns are assumed to be linear over the entire exposure sequence. Single pixel registration errors are accounted for, but not curved particle trajectories. To minimize pixel registration error, circular particle images must be recorded, not particle streaks. In high velocity flows $(>10 \mathrm{~m} / \mathrm{s})$, either fast response cameras, and/or high power, high repetition rate pulsed illumination must be used to record high quality particle images. The resolved particle images are more easily processed to determine the particle image centroids than processing a recorded particle streak. To insure high quality $2-D$ velocity vector maps, with few false identifications, five or more exposures are recommended. The more exposures used, the lower the probability of false identifications. The limit on the number of exposures used in the processing is given by the maximum tolerable particle trajectory curvature. By operating within the bounds of these limitations, particle displacement velocities can be measured across a 2-D planar region of a flow, with reasonable accuracy $(2-18 \%)$.

\section{CONCLUSIONS}

The Vector Scanning technique has many desirable qualities for a data reduction technique. The use of the particle time history information facilitated high speed processing and unambiguous velocity vector direction information. The time history information was obtained using an electronic camera to record the particle exposures. The VS algorithm can be modified to accommodate peculiarities in the way in which the data are recorded, or characteristics unique to the recorded flow field. The accuracy of the technique is inversely related to the number of pixels spanned by the particle displacement record. The maximum velocities measurable with this technique are ultimately limited by the CCD/frame-grabber board frame acquisition rate. Higher speed and higher resolution frame acquisition techniques will increase the dynamic range and precision of the processed data. The VS technique does not require specialized array processing hardware, nor are any Fourier transforms required to process the data. The processing time is directly proportional to the $\mathrm{PC}$ clock speed, and can be further reduced by using higher clock speed computers. Future work will concentrate on incorporating an intelligent decision making capability for minimizing the false identification rate, and explore the potential of using high speed, low light level electronic recording systems for recording high velocity air flows.

\section{REFERENCES}

[1] Adrian, R. J., Yao, C. S.;"Development of Pulsed Laser Velocimetry (PLV) for Measurement of Turbulent Flow", Eighth Biennial Symposium on Turbulence, U. Missouri Rolla, J. L. Kakin and G. K. Patterson, Eds. Sept 26-28, 1983.

[2] Adrian, R. J., Yao, C. S.;"Pulsed Laser Technique Application to Liquid And Gaseous Flows And The Scattering Power of Seed Materials", Appl. Opt. 24, p44, 1985 .

[3] Coupland, J. M., Pickering, C. J. D., Halliwell, N. A.;"Particle Image Velocimetry: Theory of Directional Ambiguity Removal Using Holographic Image Separation". Appl. Opt. 26, p1576, 1987.

[4] Adrian, R. J.;"Image Shifting Technique to Resolve Directional Ambiguity in Double-Pulsed Velocimetry", Appl. Opt. 25, p3855, 1986.

[5] Van Trees, H. L.;Detection, Estimation, and Modulation Theory. Part 1., Wiley Publishers, New York, 1968 .

[6] Wernet, M. P.;"A New Data Reduction Technique for Pulsed Laser Velocimetry", Ph.D. Thesis, Case Western Reserve University, May 1989.

[7] Kamotani, Y., Ostrach, S.;"Design of a Thermocapillary Flow Experiment in Reduced Gravity", AIAA-86-0200, $24^{\text {th }}$ Aerospace Sciences Meeting, Reno, Nevada, Jan., 1986. 




FIgURE 1. - VECTOR SCANNING TWO STEP PROCESSING ALGORITHM. PROCESSING STEP 1 PRODUCES THE TIME HISTORY CODED IMAGE FRAME READY FOR ANALYSIS BY THE VECTOR SCANNING PROGRAM IN STEP 2. THE OUTPUT OF STEP 2 IS THE 2-D VELOCITY VECTOR MAP. 


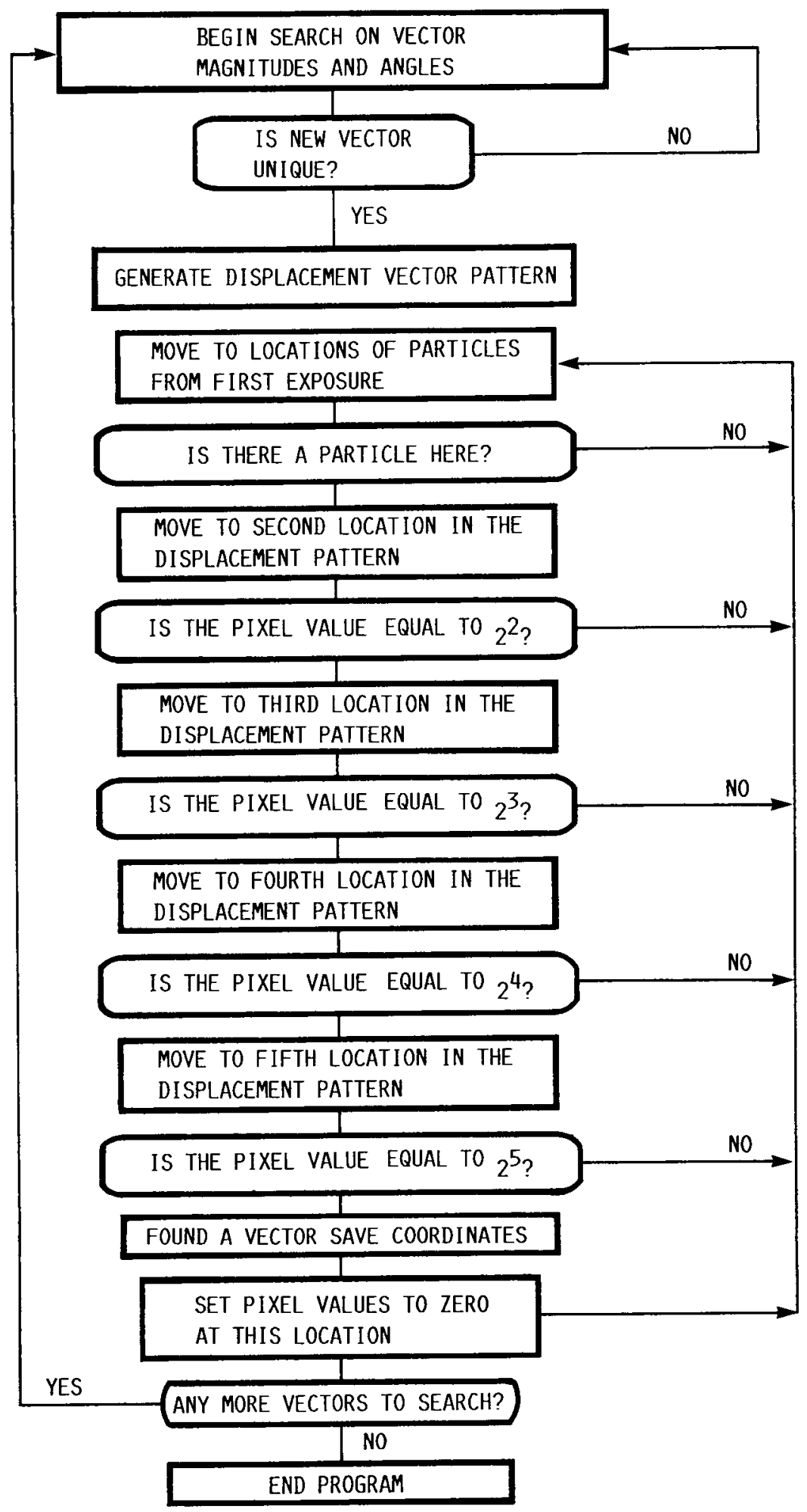

FIGURE 2. - VECTOR SCANNING PROGRAM FLOW CHART. THE PROGRAM USES THE TIME HISTORY INFORMATION TO REDUCE THE NUMBER OF FALSE CORRELATIONS AND INCREASE THE PROCESSING SPEED. 

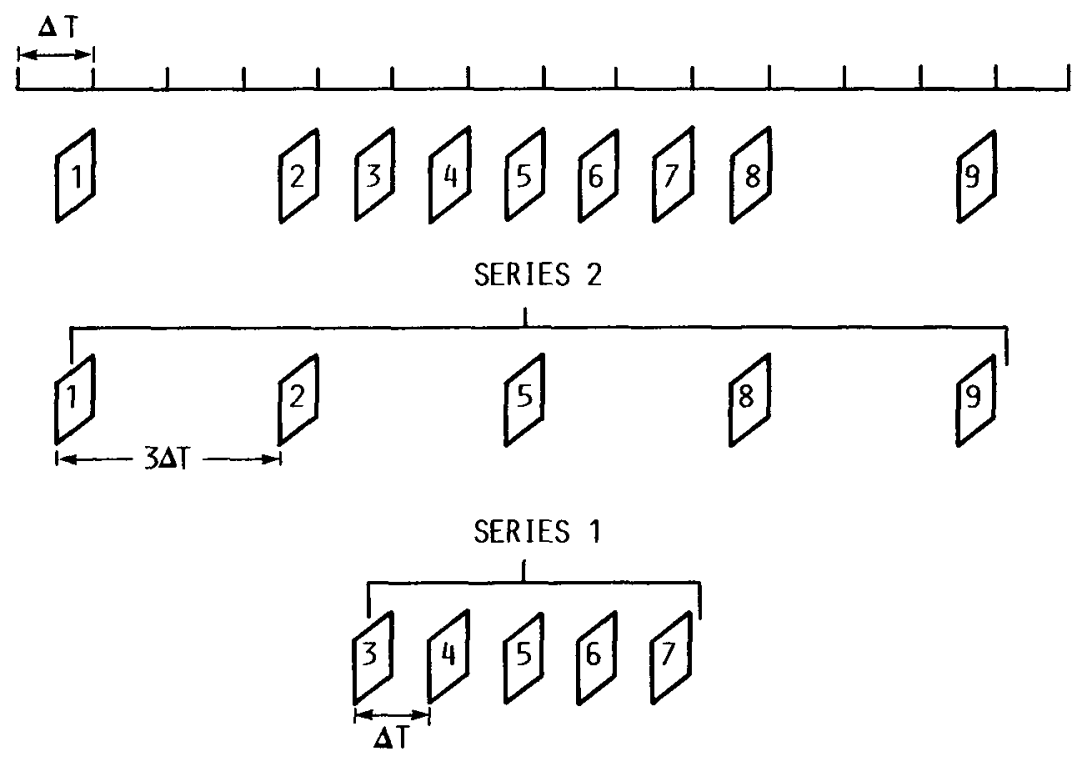

FIGURE 3. - EXTENDED RANGE FRAME RECORDING SCHEME. USING 9 FRAMES. TWO DATA SET SERIES ARE CONSTRUCTED. SERIES 1 USES FRAME \#S 3, 4. 5, 6, 7. AND SERIES 2 USES FRAME \#S 1, 2, 5, 8, 9. THE INTER-FRAME TIME FOR SERIES 1 IS $\Delta T$. SERIES 2 HAS AN INTER-FRAME TIME OF $3 \cdot \Delta T$. BOTH SERIES ARE CENTERED ABOUT, AND USE FRAME \#5.

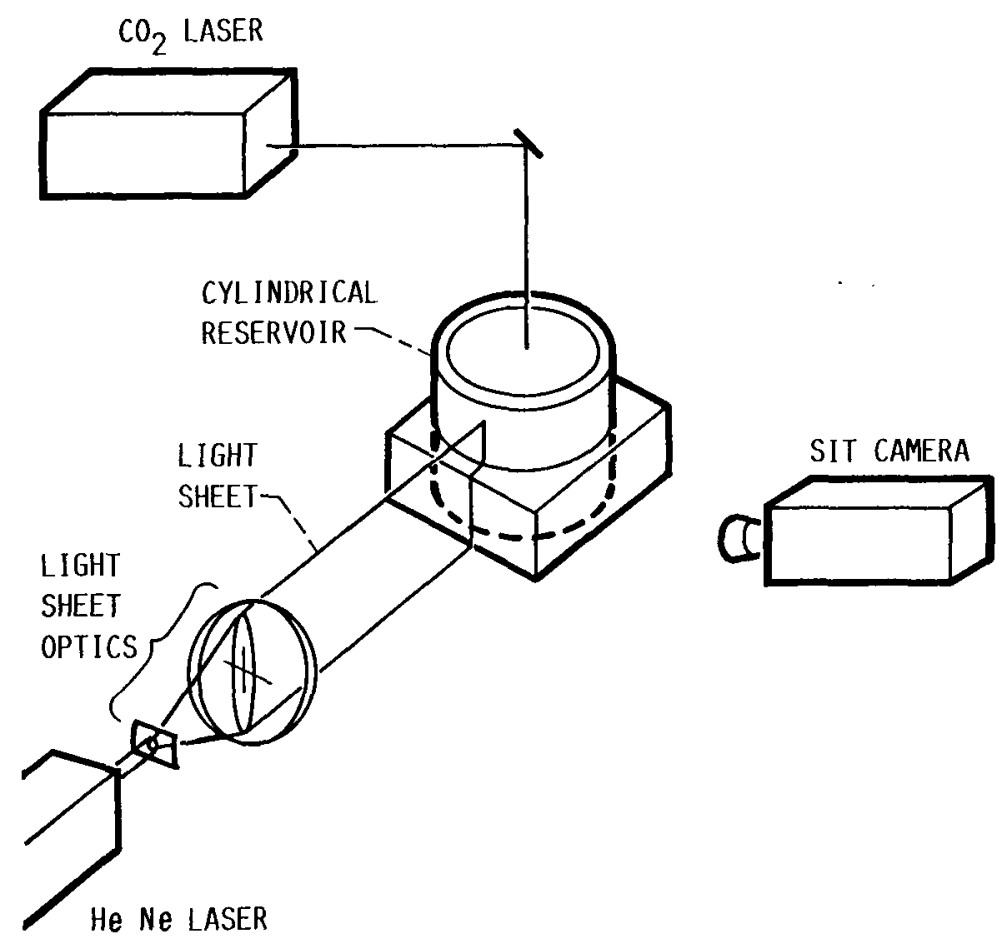

FIGURE 4. - SCHEMATIC DRAWING OF THE SURFACE TENSION DRIVEN CONVECTION SETUP. THE FLUID SURFACE IS HEATED FROM ABOVE BY $\mathrm{A} \mathrm{CO}_{2}$ LASER BEAM. THE MOTION OF THE PARTICLES IS RECORDED USING THE LASER LIGHT SHEET AND SIT CAMERA. 


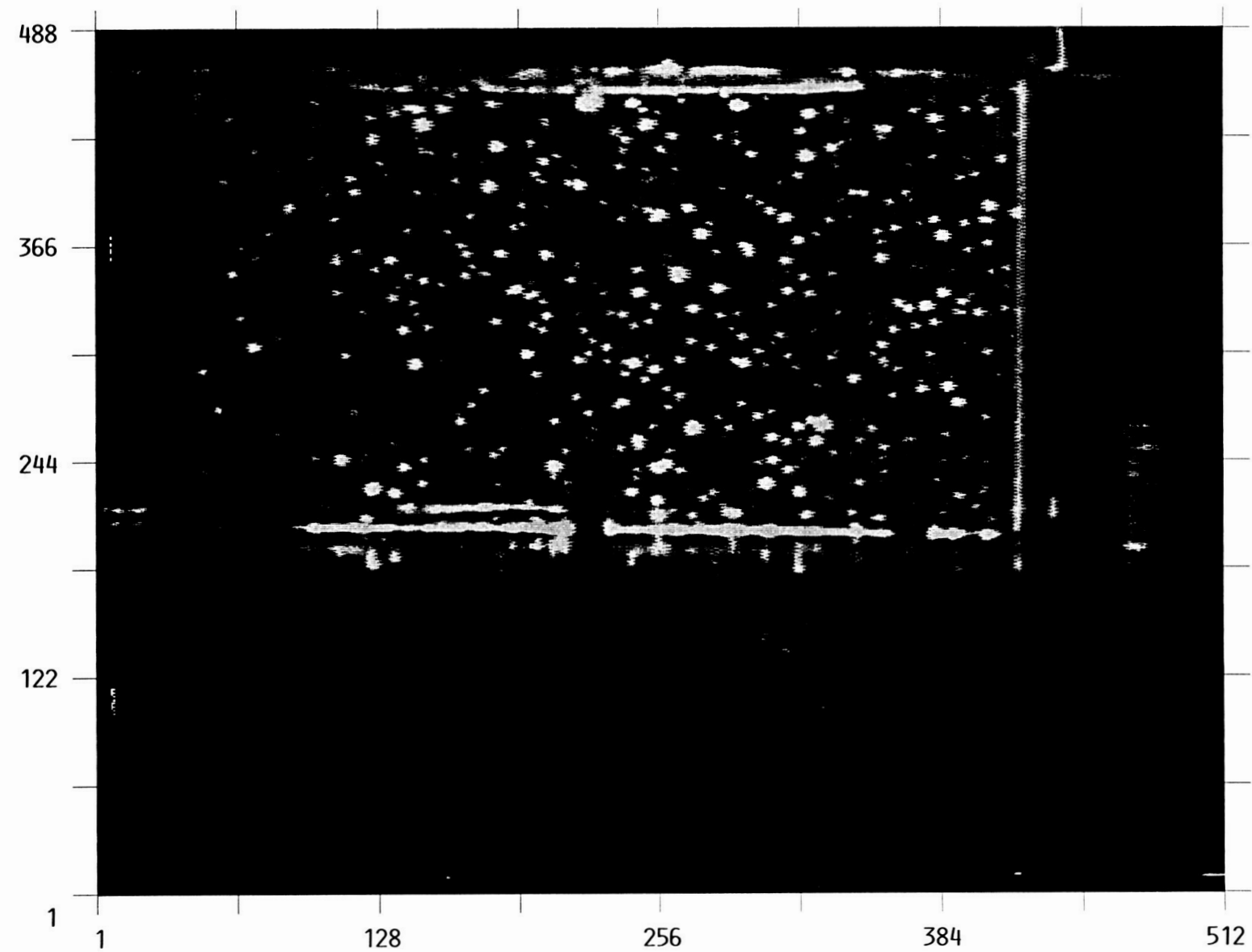

FIGURE 5. - 16 GREY LEVEL REPRODUCTION OF A SINGLE FRAME FROM THE STDC 9 FRAME SEQUENCE. FLARE LIGHT IS SCATTERED FROM THE EDGES OF THE RESERVOIR. THE TEST CELL DOES NOT FILL THE FIELD OF VIEW, WHICH IS $200 \times 200$ MM. 


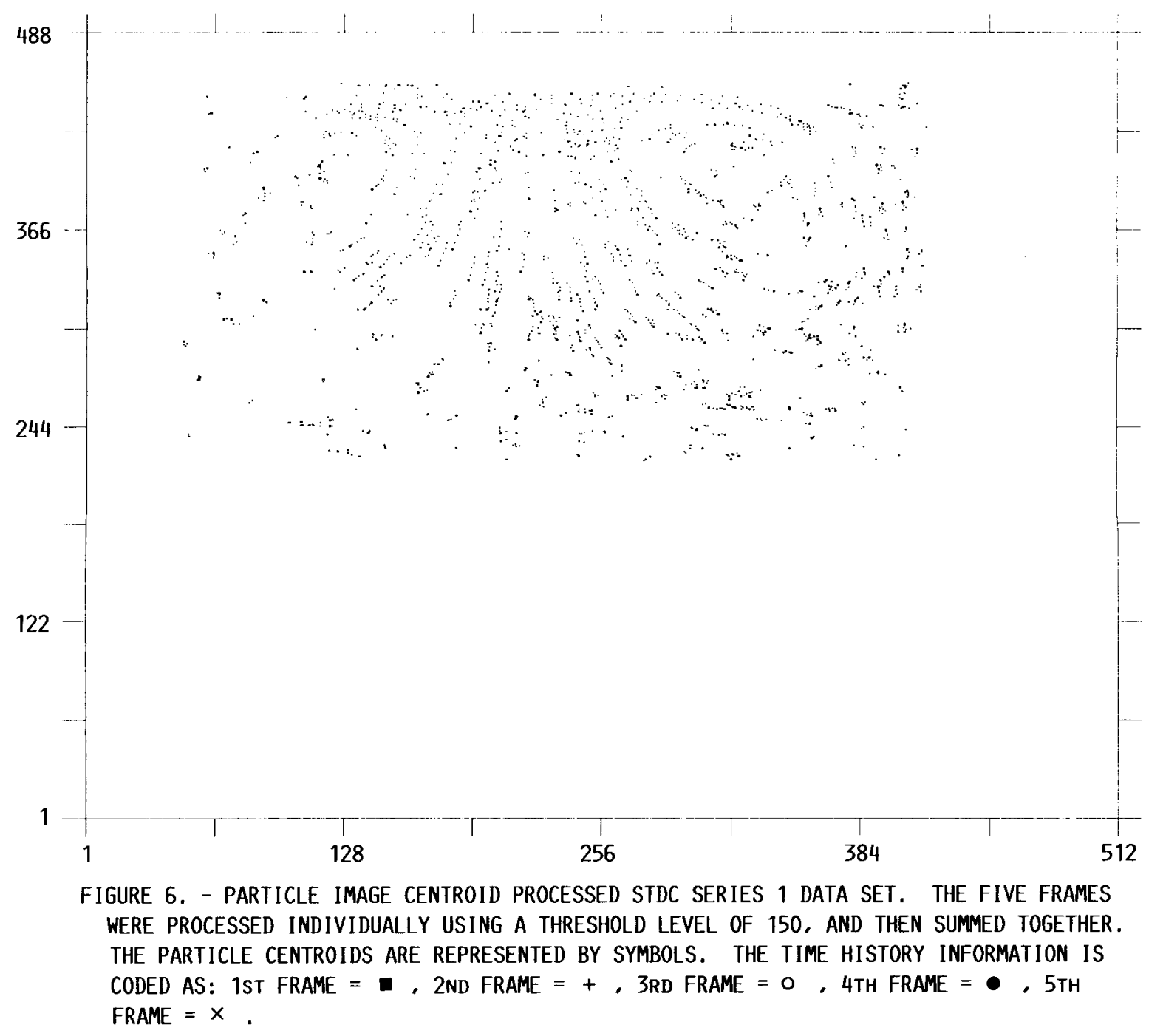




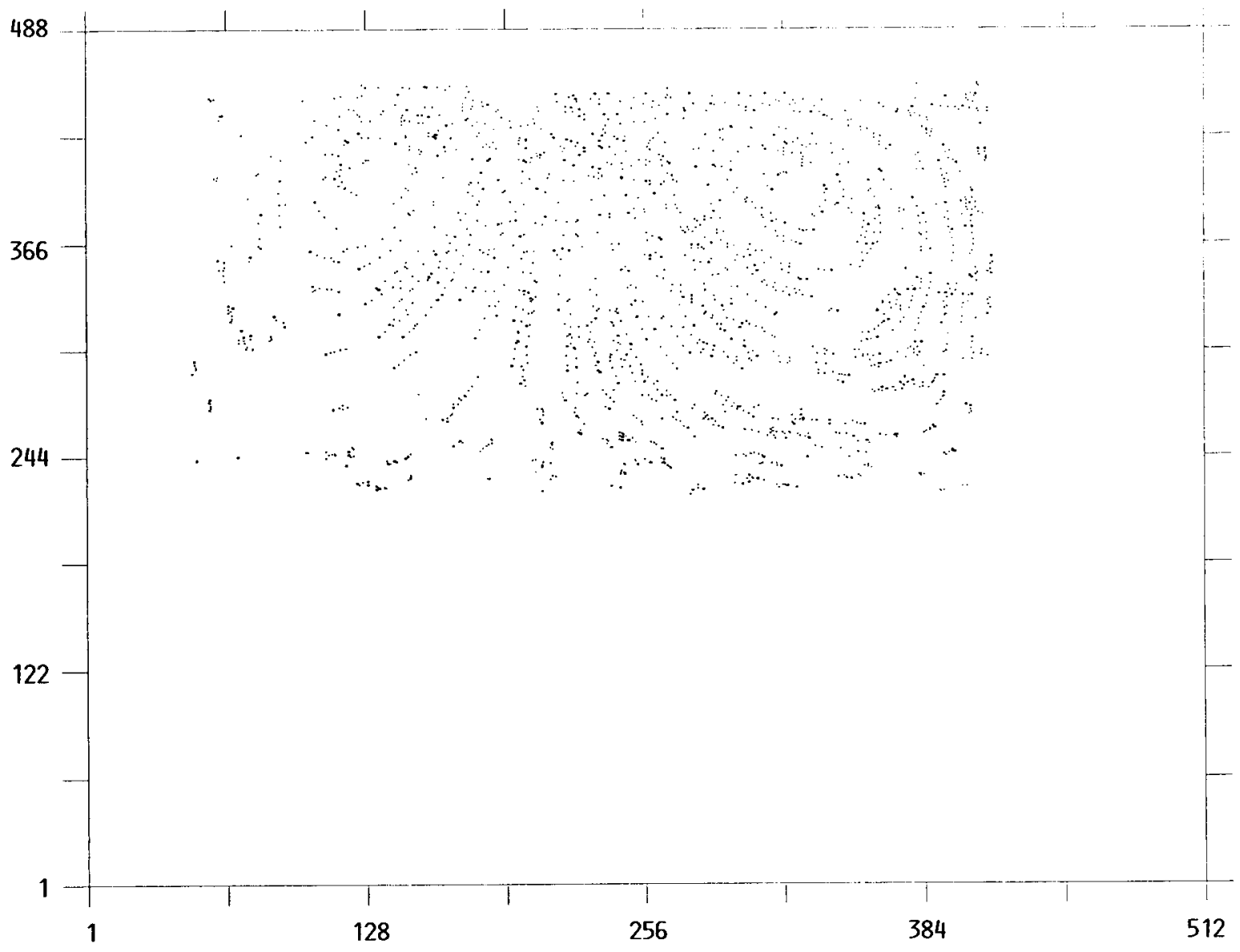

FIGURE 7. - PARTICLE IMAGE CENTROID PROCESSED STDC SERIES 2 DATA SET. THE FIVE FRAMES WERE PROCESSED INDIVIDUALLY USING A THRESHOLD LEVEL OF 150. AND THEN SUMMED TOGETHER. THE PARTICLE CENTROIDS ARE REPRESENTED BY SYMBOLS. THE TIME HISTORY INFORMATION IS CODED AS: 1ST FRAME $=0$. 2ND FRAME $=+$, 3RD FRAME $=0,4$ TH FRAME $=0.5$ TH FRAME $=x$. 


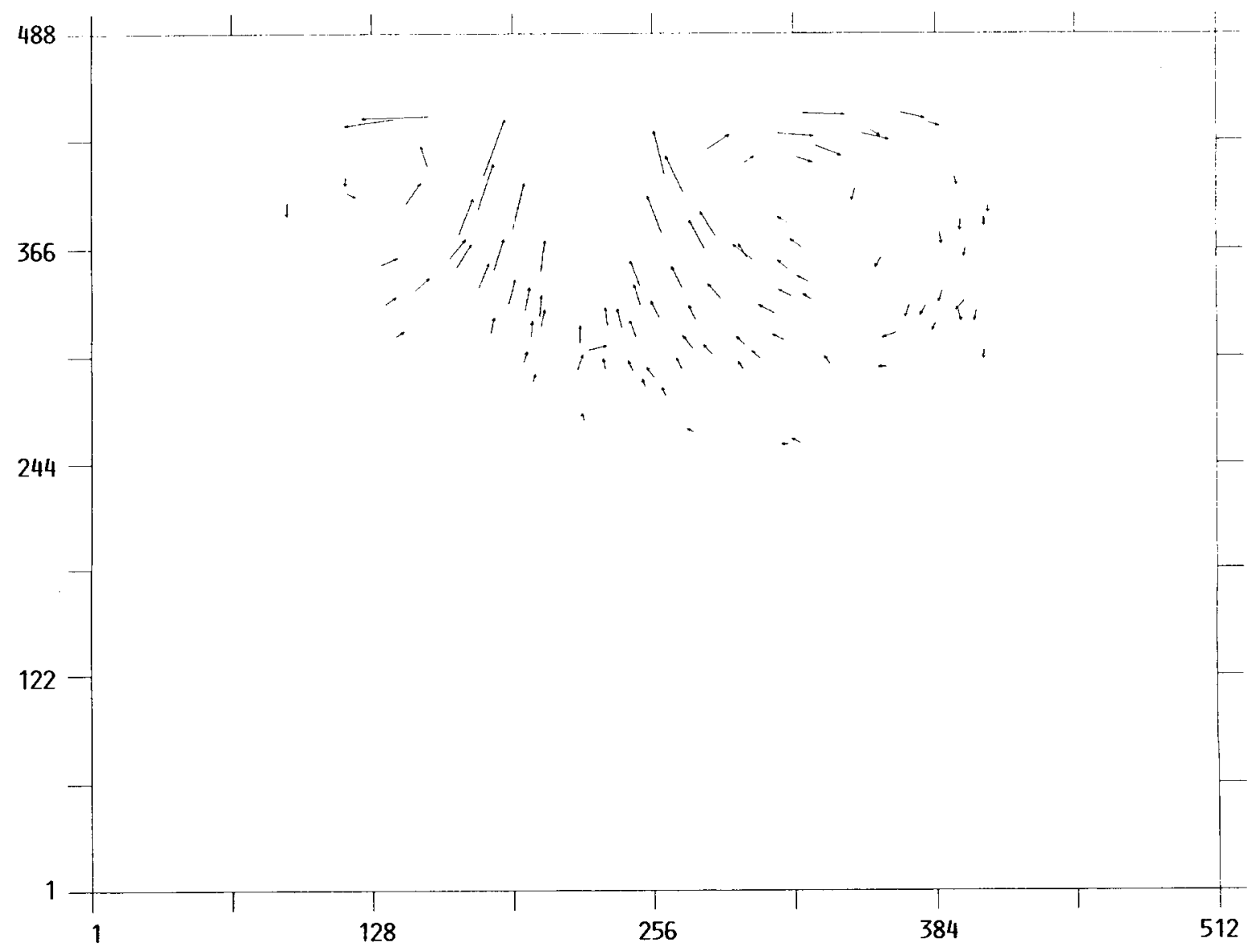

FIGURE 8. - VS PROCESSED OUTPUT VELOCITY VECTOR MAP FOR SERIES 1. THE DETECTED VELOCITY VECTORS REVEAL THE HIGH VELOCITY CORE FLOW NEAR THE HEATING POINT. THE MAXIMUM AND MINIMUM MEASURED VELOCITIES WERE $20.5 \mathrm{mM} / \mathrm{s}$ AND $2.5 \mathrm{mM} / \mathrm{s}$ RESPECTIVELY. 


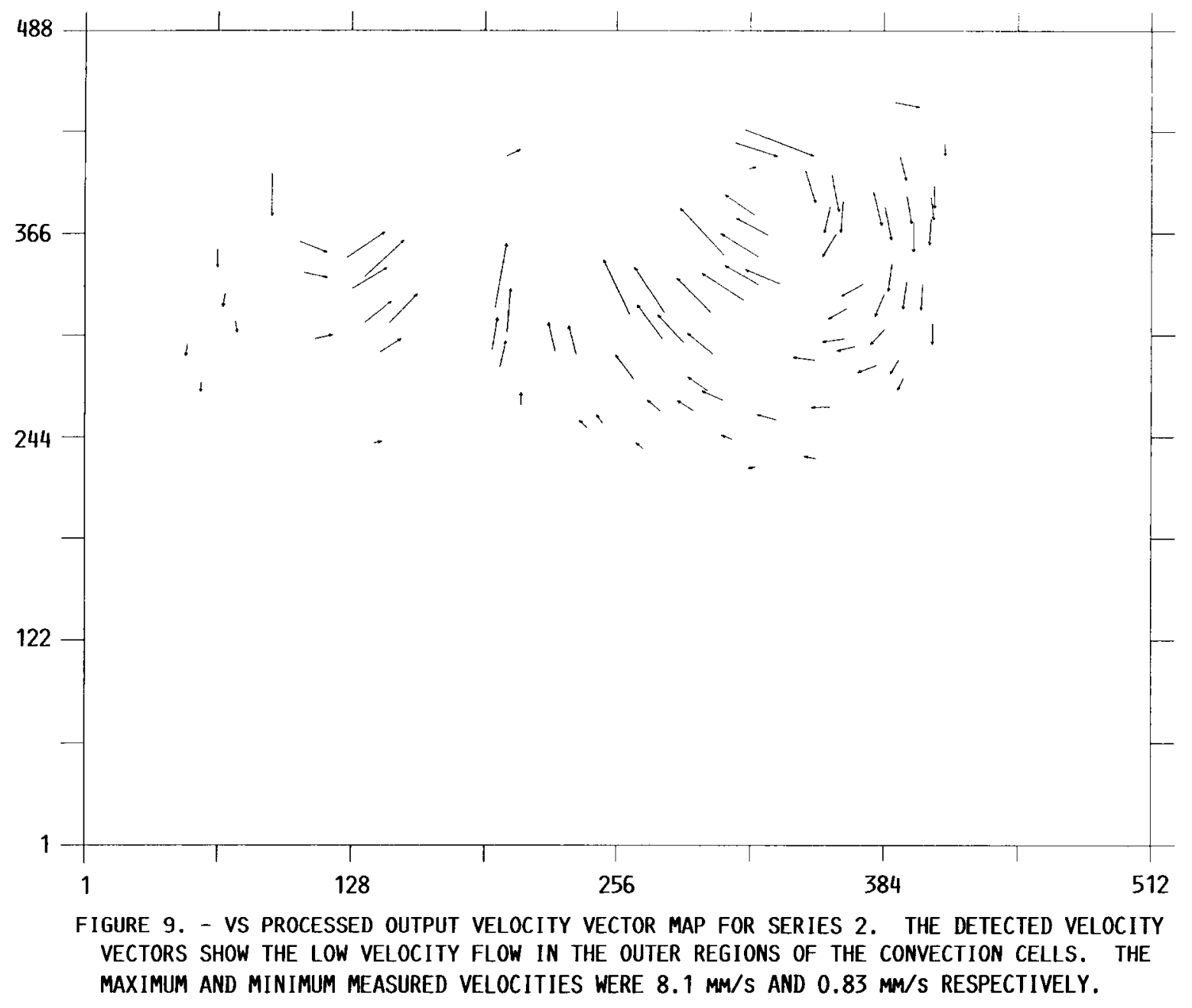




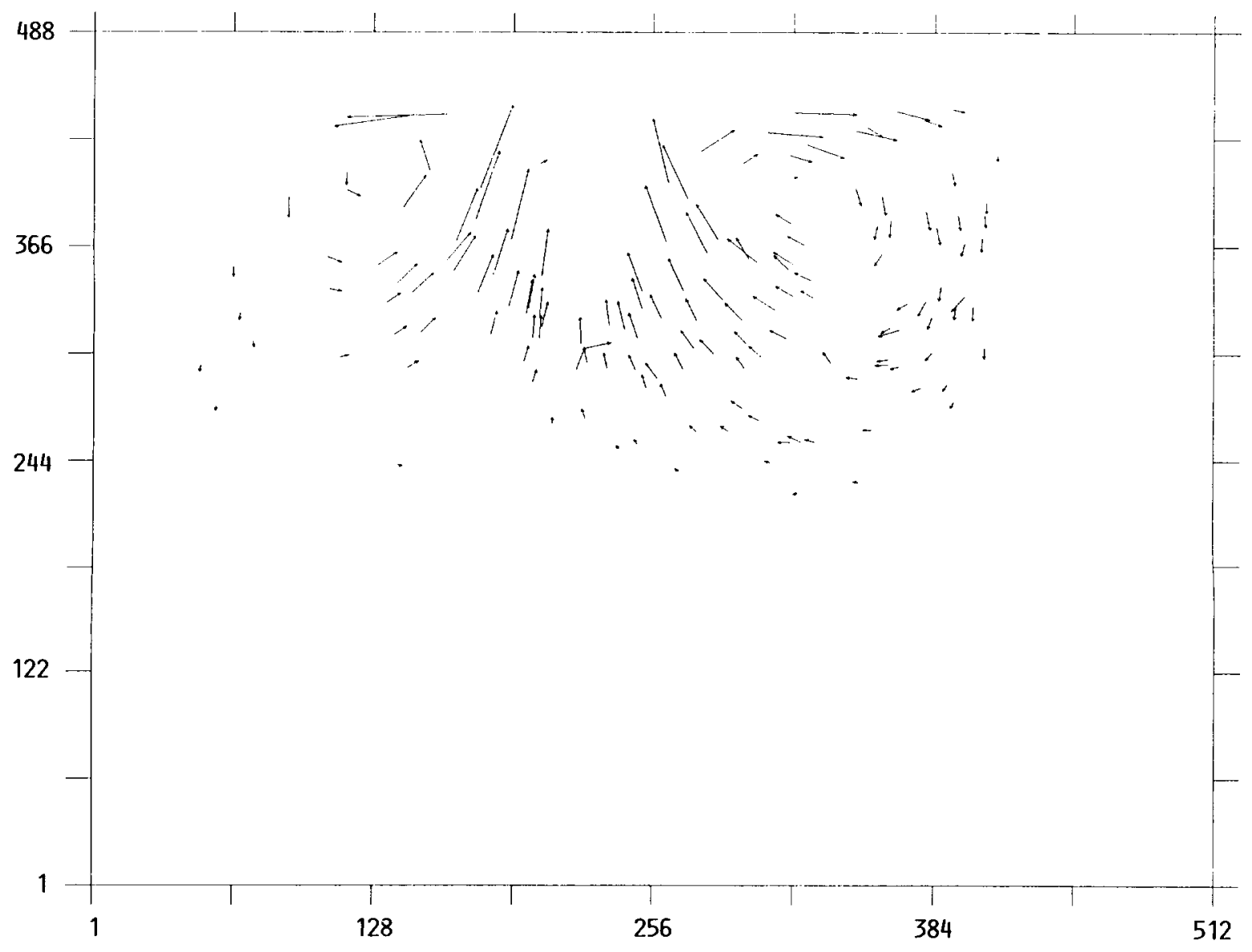

FIGURE 10. - COMBINED VELOCITY VECTOR MAP OF DATA FROM SERIES 1 AND SERIES 2 VECTOR MAPS. A HIGHER DYNAMIC RANGE VELOCITY VECTOR MAP HAS BEEN PRODUCED. THE MAXIMUM AND MINIMUM VELOCITIES ARE NOW $20.5 \mathrm{mM} / \mathrm{s}$ AND $0.83 \mathrm{mM} / \mathrm{s}$ RESPECTIVELY. THE VELOCITY VECTOR MAGNITUDE DYNAMIC RANGE IS 25:1. 


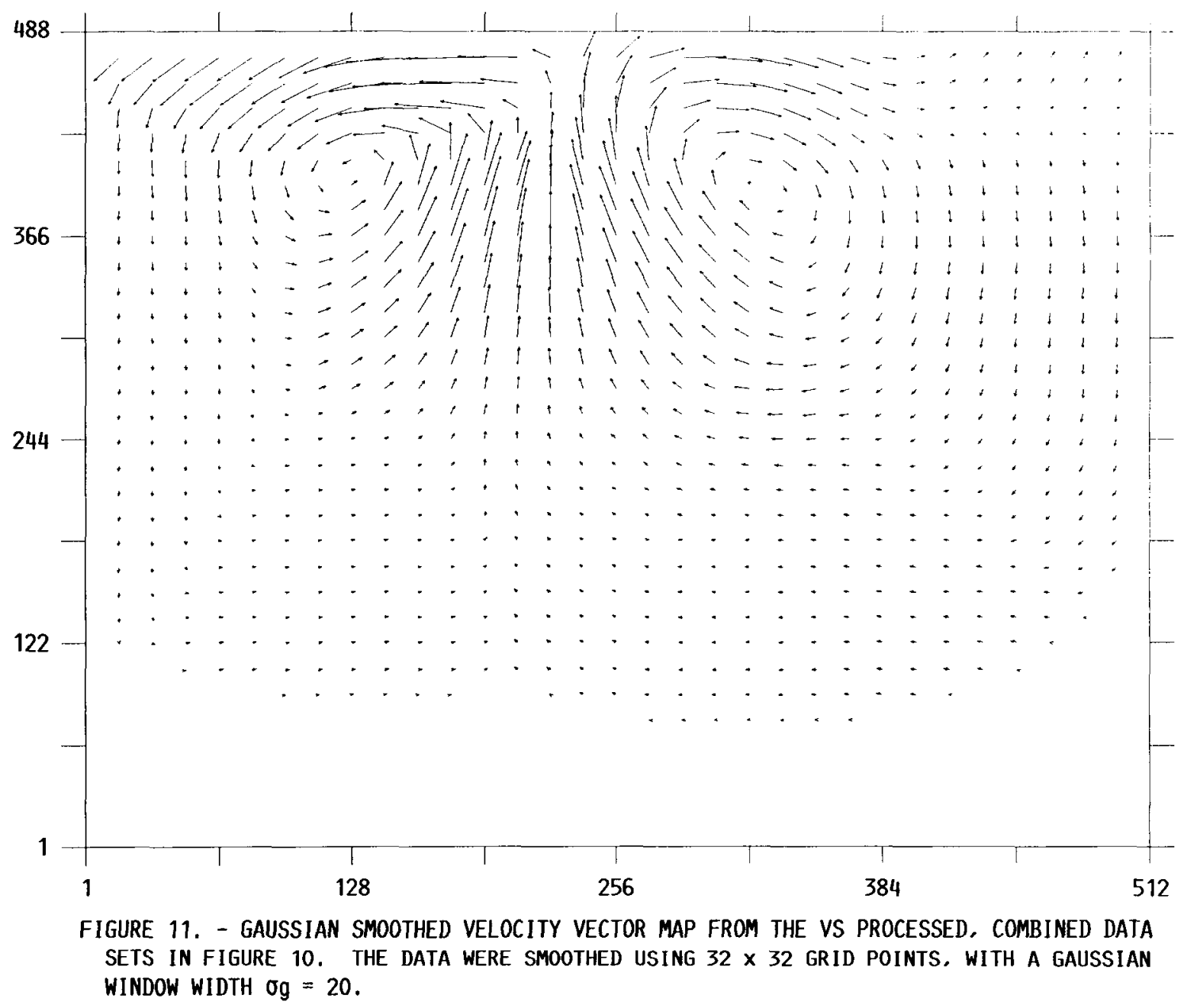




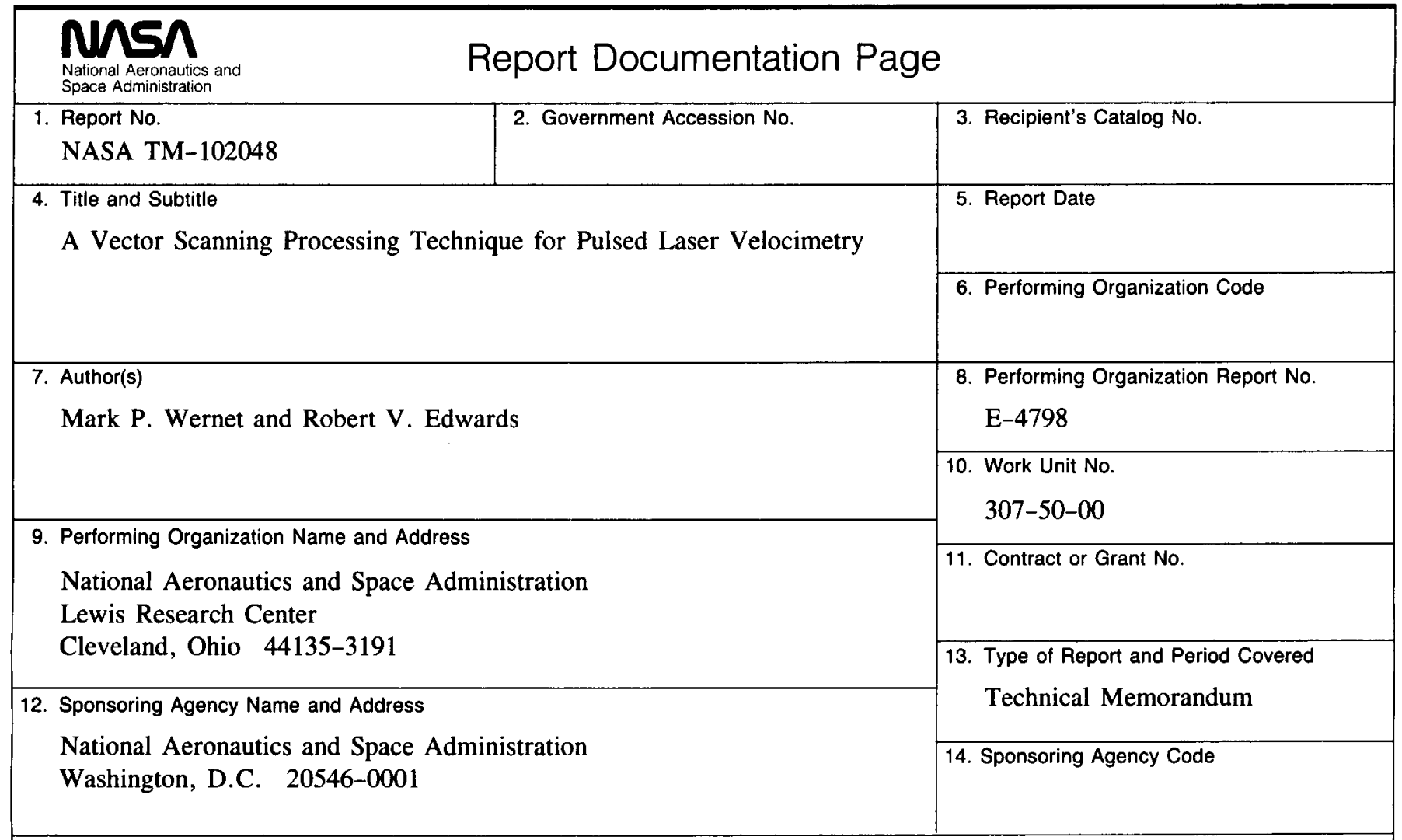

15. Supplementary Notes

Prepared for the 13th International Congress on Instrumentation in Aerospace Simulation Facilities (ICIASF) sponsored by the Institute of Electrical and Electronics Engineers, Göttingen, West Germany, September 18-21, 1989. Mark P. Wernet, NASA Lewis Research Center; Robert V. Edwards, Case Western Reserve University, Dept. of Chemical Engineering, Cleveland, Ohio 44106.

16. Abstract

Pulsed laser sheet velocimetry yields nonintrusive measurements of two-dimensional velocity vectors across an extended planar region of a flow. Current processing techniques offer high precision $(1 \%)$ velocity estimates, but can require several hours of processing time on specialized array processors. Under some circumstances, a simple, fast, less accurate $(\sim 5 \%)$, data reduction technique which also gives unambiguous velocity vector information is acceptable. In this work, we examine a direct space domain processing technique. The direct space domain processing technique was found to be far superior to any other techniques known, in achieving the objectives listed above. It employs a new data coding and reduction technique, where the particle time history information is used directly. Further, it has no $180^{\circ}$ directional ambiguity. A complex convection vortex flow was recorded and completely processed in under 2 minutes on an 80386 based PC, producing a 2-D velocity vector map of the flow field. Hence, using this new space domain Vector Scanning (VS) technique, pulsed laser velocimetry data can be reduced quickly and reasonably accurately, without specialized array processing hardware.

17. Key Words (Suggested by Author(s))

Particle imaging velocimetry

Full field measurements

Light sheet
18. Distribution Statement

Unclassified - Unlimited

Subject Category 35
19. Security Classif. (of this report)

Unclassified
20. Security Classif. (of this page)

Unclassified
21. No of pages

18
22. Price"

A03 